\title{
ChPT loops for the lattice: pion mass and decay constant, HVP at finite volume and $n \bar{n}$-oscillations
}

\author{
Johan Bijnens ${ }^{1, \star}$ \\ ${ }^{1}$ Department of Astronomy and Theoretical Physics, Lund University, Sölvegatan 14A, SE22362 Lund, Swe- \\ den
}

\begin{abstract}
I present higher loop order results for several calculations in Chiral perturbation Theory. 1) Two-loop results at finite volume for hadronic vacuum polarization. 2) A three-loop calculation of the pion mass and decay constant in two-flavour ChPT. For the pion mass all needed auxiliary parameters can be determined from lattice calculations of $\pi \pi$-scattering. 3) Chiral corrections to neutron-anti-neutron oscillations.
\end{abstract}

\section{Introduction}

This talk presents a number of calculations done using Chiral Perturbation Theory (ChPT) that should be useful for lattice calculations. The three parts that will be discussed are the vector-two-point function at two-loop order including partial quenching, twisting and finite volume. A preliminary version of the paper [1] can be found in the thesis by Johan Relefors [2]. The second part is about the first full three-loop calculation in mesonic ChPT, the pion mass and decay constant in the twoflavour case [3]. The third part is about the construction of ChPT operators for neutron-antineutron oscillations and the one-loop calculation of chiral and finite volume corrections $[4,5]$. These three parts are independent of each other, the common ground is that they all use ChPT. An introduction to ChPT for lattice practitioners is [6].

\section{The vector two-point function and HVP}

This work was done in collaboration with Johan Relefors. The main reason to consider this quantity is that the lowest order hadronic vacuum polarization contribution to the muon anomaly $a_{\mu}=\left(g_{\mu}-2\right) / 2$ can be obtained from

$$
a_{\mu}^{\mathrm{LO}, \mathrm{HVP}}=\int_{0}^{\infty} d Q^{2} f\left(Q^{2}\right)\left[\Pi_{e e}^{(1)}\left(Q^{2}\right)-\Pi_{e e}^{(1)}(0)\right] .
$$

The function $f\left(Q^{2}\right)$ is well known. The two-point function of vector currents is:

$$
\Pi_{a b}^{\mu v}(q) \equiv i \int d^{4} x e^{i q \cdot x}\left\langle T\left(j_{a}^{\mu}(x) j_{a}^{\nu^{\dagger}}(0)\right)\right\rangle \quad \quad \Pi_{a b}^{\mu \nu}=\left(q^{\mu} q^{v}-q^{2} g^{\mu \nu}\right) \Pi_{a b}^{(1)} .
$$

^e-mail: bijnens@thep.lu.se 

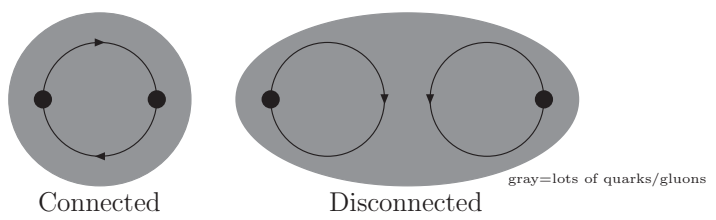

Figure 1. Connected (left) and disconnected (right) diagram(s) for the two-point function. Lines are valence quarks in a sea of quarks and gluons. Figure from [7].

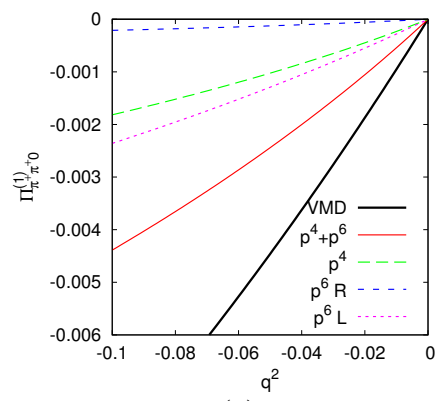

(a)

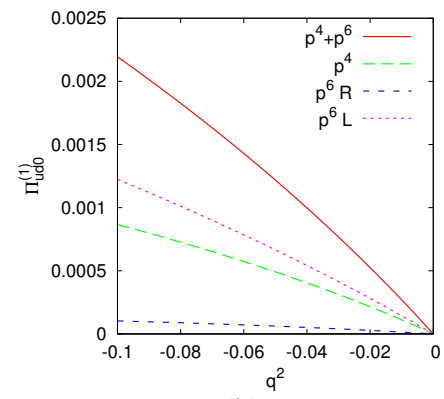

(b)

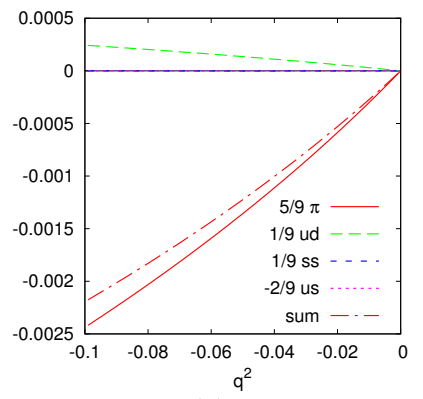

(c)

Figure 2. The vector two-point functions. (a) The disconnected part $\hat{\Pi}_{\pi^{+} \pi^{+} 0}^{(1)}$ (b) The disconnected part $\hat{\Pi}_{u d 0}^{(1)}$ (c) Various contributions to the electromagnetic $\hat{\Pi}_{e e}^{(1)}$. Figures from [7].

The last equation is valid in infinite volume for the conserved currents we use here:

$$
j_{\pi^{+}}^{\mu}=\bar{d} \gamma^{\mu} u \quad j_{u}^{\mu}=\bar{u} \gamma^{\mu} u, \quad j_{d}^{\mu}=\bar{d} \gamma^{\mu} d, \quad j_{s}^{\mu}=\bar{s} \gamma^{\mu} s, \quad j_{e}^{\mu}=(2 / 3) \bar{u} \gamma^{\mu} u-(1 / 3) \bar{d} \gamma^{\mu} d\left(-(1 / 3) \bar{s} \gamma^{\mu} s\right) .
$$

\subsection{Connected versus disconnected}

In lattice calculations there is a connected and disconnected part shown schematically in figure 1 . The disconnected part is often more difficult to calculate on the lattice so an analytic understanding of the relative sizes is very useful. This was done in ChPT at one-loop in [8] where a ratio of 1/10 was found in two-flavour ChPT. In [7] the argument was extended to two-loop order. At that order contributions from singlet vector current operators start contributing that break the ratio of $1 / 10$, however loops with the singlet current only start at even higher order. The latter is the reason for the factor $1 / 10$ as explained in [7] and in [9]. An estimate of that contribution using vector-meson-dominance (VMD) and the two-loop calculations gave reasonable agreement with the lattice results, many of which can be found in these proceedings.

We define the subtracted quantity

$$
\Pi_{a b 0}^{(1)}\left(q^{2}\right)=\Pi_{a b}^{(1)}\left(q^{2}\right)-\Pi_{a b}^{(1)}(0) .
$$

$a b=\pi^{+} \pi^{+}$gives the connected contribution for a single quark current and $a b=u d$ the disconnected part. The electromagnetic (two-flavour) case is given by $\hat{\Pi}_{e e}^{(1)}=(5 / 9) \hat{\Pi}_{\pi^{+} \pi^{+}}^{(1)}+(1 / 9) \hat{\Pi}_{u d}^{(1)}$. The results are shown in figure 2. The connected part shown in (a) has the VMD part as the largest contribution, but loops at $p^{6}$ are larger than those at $p^{4}$. This is especially due to the diagrams involving $L_{9}^{r}$. The disconnected part shown in (b) is for the loops with pions exactly $-1 / 2$ the connected part, as followed from the two-flavour singlet argument. There are small corrections from Kaon loops. In (c) we show the parts for the electromagnetic case. One can see that the strange quark current contributions are really small. A comparison with lattice data can be found in [7]. 


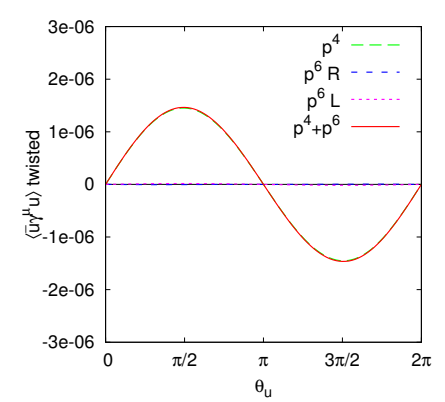

(a)

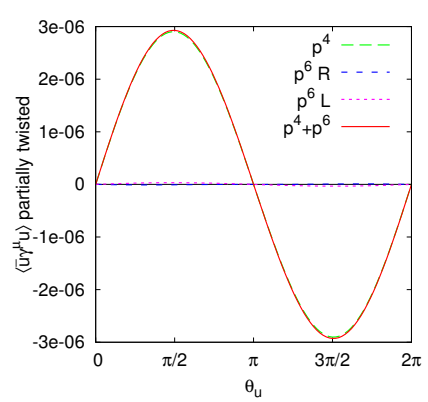

(b)
Figure 3. The vector vacuum expectation value as a function of twist angle. (a) Fully (b) Partially twisted.

\subsection{Twisting and finite volume}

The finite volume calculation at one-loop was done in $[10,11]$ and found to agree well with lattice data. Here we discuss the extension to two-loop order. Twisted boundary conditions are useful since on a lattice with periodic boundary conditions momenta are $p^{i}=2 \pi n^{i} / L$ with $n^{i}$ integer. Only a few different values of low $q^{2}$ are thus directly accessible. Putting a constraint on a quark field in some directions $q\left(x^{i}+L\right)=\exp \left(i \theta_{q}^{i}\right) q\left(x^{i}\right)$ changes the momenta to $p^{i}=\theta^{i} / L+2 \pi n^{i} / L$ allowing many more $q^{2}$. This can also be done only for the valence quarks (partial twisting). However, this twisting changes the Ward identities [10,12]. The underlying current is still conserved but now the vector current can have a vacuum expectation value changing the Ward identity to

$$
q_{\mu} \Pi_{\pi^{+} \pi^{+}}^{\mu v}=\left\langle\bar{u} \gamma^{v} u-\bar{d} \gamma^{v} d\right\rangle .
$$

Partially quenched and twisted ChPT at one and two-loop satisfy this. The numerical size of the effect is quite small [1]. The vacuum expectation value as a function of the twist angle for a fully and partially twisted up-quark with a twist angle in one direction only is shown in figure 3 . These are for $m_{\pi} L=4$, the $p^{6}$ corrections are really small. The numbers should be compared with the scalar vacuum expectation value and its finite volume correction $[1,13],\langle\bar{u} u\rangle \approx-1.210^{-2} \mathrm{GeV}^{3}$ and $\langle\bar{u} u\rangle^{V} \approx-2.410^{-5} \mathrm{GeV}^{3}$.

How large are now the corrections due to finite volume and twisting? The low-energy constants (LECs) we use are those of [14]. The calculations were done in [1]. Numerical results for the finite volume corrections at $m_{\pi} L=4$ as a function of $q^{2}$ are shown in figure 4 . These should be compared to the VMD contribution which is of order $0.005\left(q^{2} / 0.1 \mathrm{GeV}^{2}\right)$. So the finite volume correction are rather $q^{2}$ dependent but not large. In particular, the $p^{6}$-corrections are very small, very different from the infinite volume result. One can also see the difference between different ways of obtaining the same $q^{2}$ by partial twisting, in (a) it is done in a spatially symmetric fashion, in (b) only in one direction. The difference allows for checking the finite volume corrections with the same underlying lattice.

One often calculates on the lattice the spatial average over the two-point function components. This is defined in the caption of figure 5 and numerical results at order $p^{4}$ are shown in figure 5(a) and the full $p^{4}+p^{6}$ result in figure 5(b). Again, the difference between the two can be used to see check the estimates of the finite volume corrections using only one underlying lattice.

\section{The pion mass and decay constant at three-loops}

This is work done in collaboration with Nils Hermansson Truedsson [3]. The pion mass and decay constant have been calculated in ChPT before at tree-level [15], one-loop chiral logarithms [16], full 


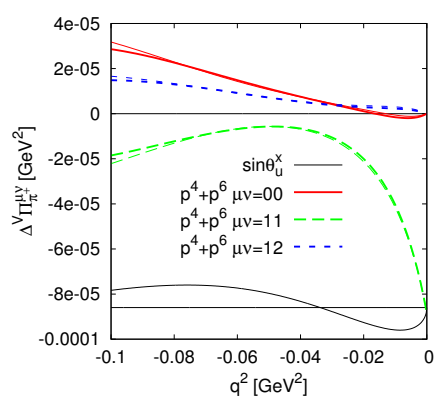

(a)

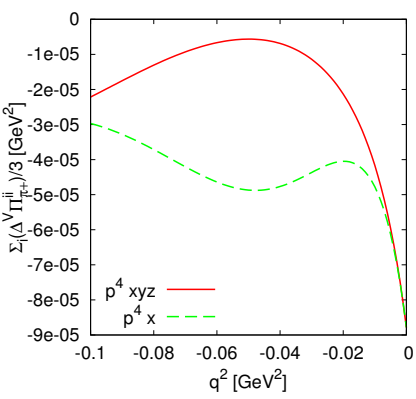

(a)

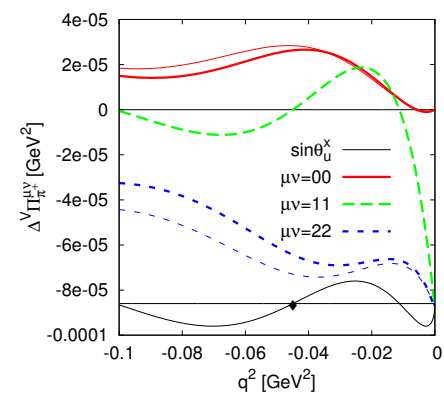

(b)

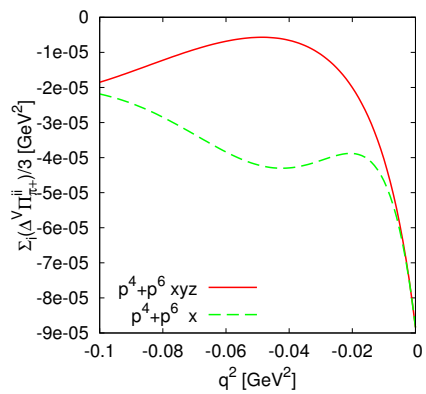

(b)
Figure 4. Finite volume corrections to some components of the two-point function $\Pi_{\pi^{+} \pi^{+}}^{\mu \nu}$. The thin lines are $p^{4}$-only, the thick lines $p^{4}+p^{6}$. (a) Spatially symmetric twisting (b) twisting in one direction only. The diamond indicates a $q^{2}$ accessible with periodic boundary conditions. Bottom curve shows $\sin \theta_{u}^{x}$, the sine of the twisting angle in the $x$-direction, they help understanding the shape of the curves. Figures from [1].

Figure 5. Finite volume corrections to the trace over spatial components $\bar{\Pi}=(1 / 3) \sum_{i=x, y, z} \Pi^{i i}$ as a function of $q^{2}$ for two different ways of twisting, spatially symmetric and in one direction only. (a) $p^{4}$ (b) $p^{4}+p^{6}$. Figures from [1].

one-loop and incidentally the proper start of ChPT [17], as well as two-loop [18-20]. Each new step introduced a number of new methods. Lowest order (LO) and chiral logs were done with current algebra. The full one-loop calculation was done by hand and by directly expanding the functional integral with the help of REDUCE. The NNLO or two-loop work was done by hand with a little help from FORM. The NNNLO result required a large amount of use of FORM [21]. The main stumbling block is really the integrals. The reduction to master integrals was done with REDUZE [22]. All master integrals are known $[23,24]$.

A calculation of this size also requires a large number of checks. The nonlocal divergences must cancel as in any field theory. We used two different parametrizations of the Lagrangians, square root and exponential, and the leading logarithms agreed with the result derived earlier [25, 26]. The diagrams are shown in figure 6.
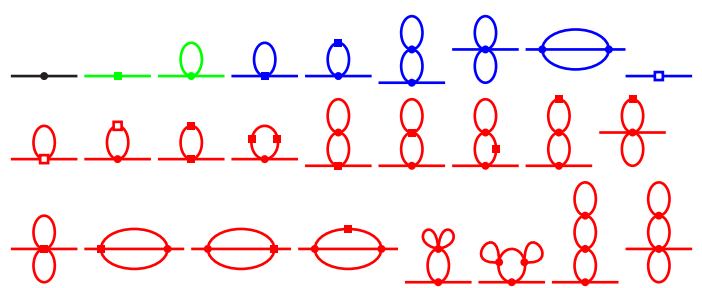

Figure 6. The diagrams for the pion mass and decay constant to NNNLO in ChPT. Figure from [3].

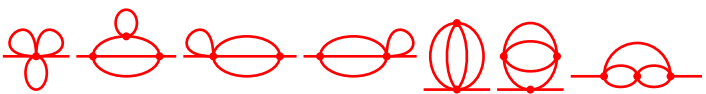

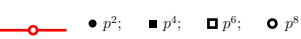




\begin{tabular}{|c||c|c|c|c|}
\hline$i j$ & $a_{i j}^{M}$ & $b_{i j}^{M}$ & $a_{i j}^{F}$ & $b_{i j}^{F}$ \\
\hline \hline 10 & 0.0028 & -0.0028 & 1.0944 & -1.0944 \\
\hline 11 & 0.5 & -0.5 & -1.0 & 1.0 \\
\hline 20 & 1.6530 & -1.6577 & -0.0473 & -1.1500 \\
\hline 21 & 2.4573 & -3.2904 & -1.9058 & 4.1388 \\
\hline 22 & 2.125 & -0.625 & -1.25 & -0.25 \\
\hline 30 & 0.4133 & -6.8035 & -244.5350 & 242.2724 \\
\hline 31 & -3.7044 & 4.2718 & -15.4989 & 28.5703 \\
\hline 32 & 17.1476 & 0.6204 & -9.3946 & -6.7751 \\
\hline 33 & 4.2917 & 5.1458 & -3.4583 & -0.4167 \\
\hline
\end{tabular}

Table 1. Numerical values of the $a_{i j}^{M, F}$ and $b_{i j}^{M, F}$ for the input parameters given in the text. Table from [3].

We can do the expansion of the physical quantities $M_{\pi}^{2}, F_{\pi}$ in terms the LO quantities $M^{2}, F(x$ expansion) or the inverse ( $\xi$-expansion). $M^{2}=2 B \hat{m}$ and $F$ are the LO pion mass and decay constant. The $x$-expansions are

$$
\begin{aligned}
& M_{\pi}^{2}=M^{2}\left\{1+x\left(a_{10}^{M}+a_{11}^{M} L_{M}\right)+x^{2}\left(a_{20}^{M}+a_{21}^{M} L_{M}+a_{22}^{M} L_{M}^{2}\right)+x^{3}\left(a_{30}^{M}+a_{31}^{M} L_{M}+a_{32}^{M} L_{M}^{2}+a_{33}^{M} L_{M}^{3}\right)\right\} \\
& F_{\pi}=F\left\{1+x\left(a_{10}^{F}+a_{11}^{F} L_{M}\right)+x^{2}\left(a_{20}^{F}+a_{21}^{F} L_{M}+a_{22}^{F} L_{M}^{2}\right)+x^{3}\left(a_{30}^{F}+a_{31}^{F} L_{M}+a_{32}^{F} L_{M}^{2}+a_{33}^{F} L_{M}^{3}\right)\right\}
\end{aligned}
$$

with $x=M^{2} /\left(16 \pi^{2} F^{2}\right)$ and $L_{M}=\log \left(M^{2} / \mu^{2}\right)$, and the $\xi$-expansions are

$$
\begin{aligned}
M^{2} & =M_{\pi}^{2}\left\{1+\xi\left(b_{10}^{M}+b_{11}^{M} L_{\pi}\right)+\xi^{2}\left(b_{20}^{M}+b_{21}^{M} L_{\pi}+b_{22}^{M} L_{\pi}^{2}\right)+\xi^{3}\left(b_{30}^{M}+b_{31}^{M} L_{\pi}+b_{32}^{M} L_{\pi}^{2}+b_{33}^{M} L_{\pi}^{3}\right)\right\} \\
F & =F_{\pi}\left\{1+\xi\left(b_{10}^{F}+b_{11}^{F} L_{\pi}\right)+\xi^{2}\left(b_{20}^{F}+b_{21}^{F} L_{\pi}+b_{22}^{F} L_{\pi}^{2}\right)+\xi^{3}\left(b_{30}^{F}+b_{31}^{F} L_{\pi}+b_{32}^{F} L_{\pi}^{2}+b_{33}^{F} L_{\pi}^{3}\right)\right\}
\end{aligned}
$$

where $\xi=M_{\pi}^{2} /\left(16 \pi^{2} F_{\pi}^{2}\right)$ and $L_{\pi}=\log \left(M_{\pi}^{2} / \mu^{2}\right)$. The analytical values for the coefficients can be found in [3]. We found that the coefficients of the logarithms for the mass at order $p^{8}$ can all be written in terms of coefficients obtainable from the lattice from $\pi \pi$-scattering to two-loop order.

For the numerical estimates, we use $\mu=0.77 \mathrm{GeV}, \bar{l}_{1}=-0.4, \bar{l}_{2}=4.3, \bar{l}_{3}=3.41, \bar{l}_{4}=4.51$ and the $\pi \pi$-scattering estimates from [20]. All remaining LECs have been set to zero. The resulting values for the coefficients are give in table 1 . The numerical values of $a_{30}^{F}$ and $b_{30}^{F}$ are rather large, due to a very large numerical coefficient $383293667 / 1555200 \approx 246.5$ appearing. The remaining coefficients are of natural magnitude.

The quantities (6)-(7) are shown in figure $7(\mathrm{a}-\mathrm{d})$, with the same inputs as above. $F=$ $92.2 / 1.073 \mathrm{MeV}$ is kept constant for $x$-expansions, while $F_{\pi}=92.2 \mathrm{MeV}$ is fixed for the $\xi$-expansion. $M$ and $M_{\pi}$ are varied respectively. The convergence near the physical value $M_{\pi}^{2} \approx 0.02 \mathrm{GeV}^{2}$ is excellent. The $\xi$-expansion converges better.

\section{ChPT for $n \bar{n}$-oscillations}

This work was done in collaboration with Erik Kofoed. The baryon asymmetry of the universe is one of the unsolved problems in particle physics. One way to solve it is via $\Delta B=2$-transitions that appear in certain GUTs without proton decay, see [27] for a review and further references. There is a proposal for a free neutron oscillation experiment at ESS in Lund that might improve the present limit by three orders of magnitude. Neutron-antineutron oscillations need an operator consisting of at least six quarks, schematically $u u d d d d$. The lowest dimension operators of this type have dimension 9 and there are 14 of them. A classification and the short-distance running to two-loop order is in [28], earlier references can be found there and in [4, 27]. 


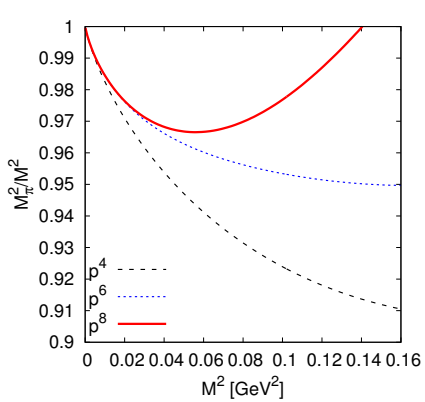

(a)

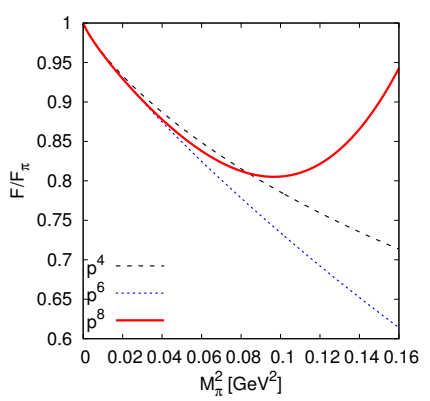

(d)

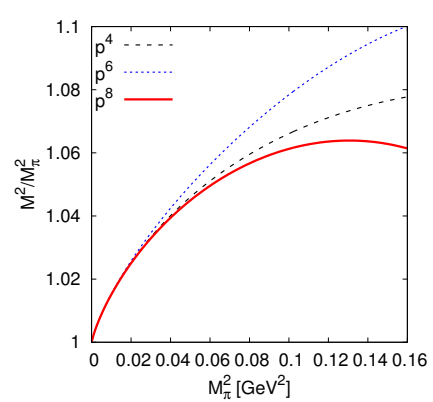

(b)

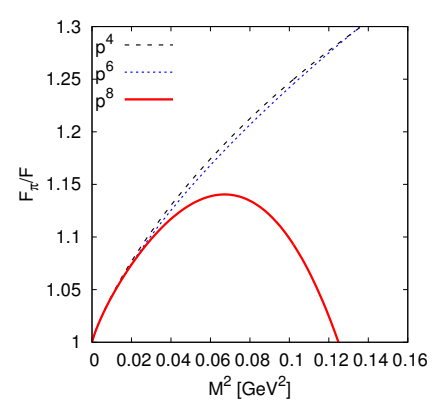

(c)

Figure 7. (a) The $x$-expansion for the mass, (b) the $\xi$-expansion for the mass, (c) the $x$-expansion for the decay constant, (d) the $\xi$-expansion for the decay constant at NLO, NNLO and NNNLO. LO is constant at 1 for all plots. Figures from [3].

There are 14 dimension 9 six-quark uudddd operators which transform under chiral symmetry $S U(2)_{L} \times S U(2)_{R}$ as $\left.\left(3_{L}, 1_{R}\right)\left(P_{1}, P_{2}, P_{3}\right),\left(3_{L}, 5_{R}\right)\left(P_{5}, P_{6}, P_{7}\right)\right),\left(7_{L}, 1_{R}\right)\left(P_{4}\right)$ and the parity conjugates $Q_{1}, \ldots, Q_{7}$. The operators $P_{5}, P_{6}, P_{7}$ belong to the same chiral multiplet, so they have the same low-energy constants, while $P_{1}, P_{2}, P_{3}$ are not related. In fact $P_{5}, P_{6}, P_{7}$ are related by isospin so chiral corrections are the same for all of these even if chiral symmetry is spontaneously broken. $n \bar{n}$ transitions are isospin 1 , so the operators $P_{4}, Q_{4}$, which are isospin 3, do not contribute.

In constructing ChPT operators we use the spurion technique. The needed spurions have two $S U(2)_{L}$ doublet indices symmetrized to make a $3_{L}$, four $S U(2)_{R}$ indices symmetrized to make a $5_{R}$ and six $S U(2)_{L}$ doublet indices to make a $7_{L}$ (and $L \leftrightarrow R$ for the parity-conjugates). We use heavy baryon ChPT way to include the nucleon field $\mathcal{N}$ with fourvelocity $v$, see e.g. [29, 30]. We need to introduce also a HBCHPT antinucleon field. This is expanding around two widely separated areas in momentum space, around $m_{N} v$ for the nucleons and $-m_{N} v$ for the antinucleons, in the relativistic fields. In HBCHPT these become independent fields. The lowest order Lagrangian becomes ${ }^{1}$

$$
\mathcal{L}_{L O}=\frac{F^{2}}{4}\left\langle u_{\mu} u^{\mu}+\chi_{+}\right\rangle+\overline{\mathcal{N}}\left(i v^{\mu} D_{\mu}+g_{A} u^{\mu} S_{\mu}\right) \mathcal{N}+\overline{\mathcal{N}^{c}}\left(i v^{\mu} D_{\mu}-g_{A} u^{\mu} S_{\mu}\right) \mathcal{N}^{c} .
$$

The definitions are the usual ChPT notation, see e.g. [29]. The fields we use are defined as

$$
\mathcal{N}=\left(\begin{array}{c}
p \\
n
\end{array}\right), \quad \quad \mathcal{N}^{c}=\left(\begin{array}{c}
n^{c} \\
-p^{c}
\end{array}\right), \quad \widetilde{\mathcal{N}^{c}}=\left(\begin{array}{c}
\overline{p^{c}} \\
n^{c}
\end{array}\right) .
$$

$p, n$ are the nucleon, $p^{c}, n^{c}$ the antinucleon HBCHPT fields. The objects in $\mathcal{N}^{i}$ in (9) transform under chiral symmetry all as $\mathcal{N}^{i} \rightarrow h\left(u, g_{L}, g_{R}\right) \mathcal{N}^{i}$ where $h\left(u, g_{L}, g_{R}\right)$ is the usual $S U(2)_{V}$ compensator transformation. With these we can now construct ChPT operators with the chiral transformation properties of the uudddd quark operators.

\footnotetext{
${ }^{1}$ This differs slightly from what was shown during the talk to have objects with simpler chiral transformations.
} 


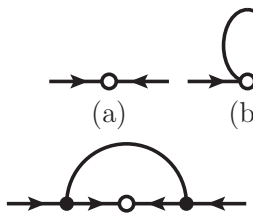

(e) (b)
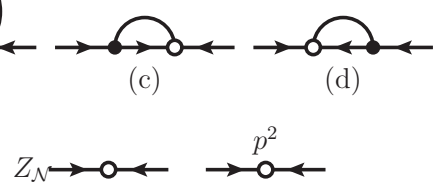

(f)

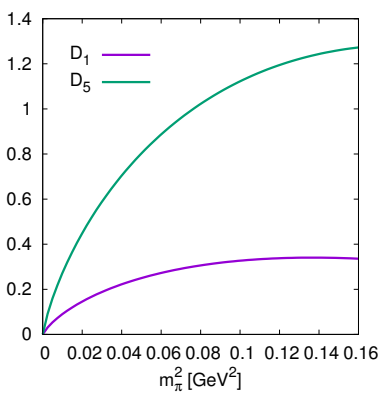

(a)

(g)

Figure 8. The $n \bar{n}$ transition diagrams to order $p^{2}$. An open dot is a vertex from the $n \bar{n}$ operators (10), a dot from the LO normal Lagrangian (8). Wave-function renormalization is indicated schematically in (f) and $p^{2} n \bar{n}$-operators in (g). A right-pointing line is a nucleon, a left-pointing line an antinucleon.

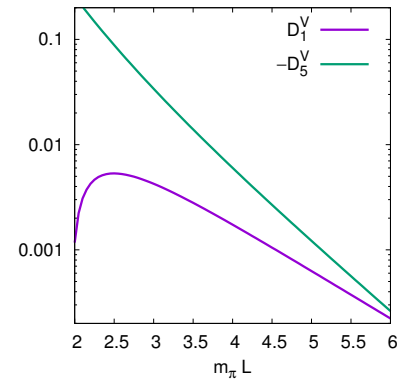

(b)
Figure 9. The numerical results of the pure loop contributions. (a) The infinite volume correction of (12) (b) The finite volume correction. Figures from [4].

The lowest order, $p^{0}$, operators are

$$
\begin{aligned}
& \left(3_{L}, 1_{R}\right): R_{i_{L} j_{L}}=\left(u^{\dagger} \widetilde{\mathcal{N}^{c}}\right)_{i_{L}}\left(u^{\dagger} \mathcal{N}\right)_{j_{L}} \\
& \left(3_{L}, 5_{R}\right): R_{i_{L} j_{L} k_{R} l_{R} m_{R} n_{R}}=\left(u \widetilde{\mathcal{N}}^{c}\right)_{k_{R}}(u \mathcal{N})_{l_{R}}\left(U i \tau^{2}\right)_{m_{R} i_{L}}\left(U i \tau^{2}\right)_{n_{R} j_{L}}
\end{aligned}
$$

and the parity-conjugates. There is no lowest order operator for $\left(7_{L}, 1_{R}\right)$. The Dirac (fermion) indices are contracted between $\mathcal{N}$ and $\widetilde{\mathcal{N}^{c}}$. The first operator for $\left(7_{L}, 1_{R}\right)$ appears at order $p^{2}$ :

$$
\left(7_{L}, 1_{R}\right), p^{2}:\left(u^{\dagger} \widetilde{\mathcal{N}^{c}}\right)_{i_{L}}\left(u^{\dagger} \mathcal{N}\right)_{j_{L}}\left(u^{\dagger} u_{\mu} u i \tau_{2}\right)_{k_{L} l_{L}}\left(u^{\dagger} u_{\mu} u i \tau_{2}\right)_{m_{L} n_{L}}
$$

The operators at order $p^{1}$ do not contribute to $n \bar{n}$, at most via loops so starting only at $p^{3}$. At higher orders there are very many operators. A partial list at order $p^{2}$ can be found in [5].

The loop corrections can now be calculated. The diagrams are shown in figure 8 .

Due to isospin the three $\left(3_{L}, 1_{R}\right)$ and the three $\left(3_{L}, 5_{R}\right)$ each have the same relative loop corrections. The expressions for the relative corrections, i.e. multiply the lowest order result by $1+D_{i}$, are

$$
\begin{aligned}
& D_{1}=\frac{m_{\pi}^{2}}{16 \pi^{2} F^{2}}\left[\left(-1-\frac{3 g_{A}^{2}}{2}\right) \log \frac{m_{\pi}^{2}}{\mu^{2}}-g_{A}^{2}\right], \\
& D_{5}=\frac{m_{\pi}^{2}}{16 \pi^{2} F^{2}}\left[\left(-7-\frac{3 g_{A}^{2}}{2}\right) \log \frac{m_{\pi}^{2}}{\mu^{2}}-g_{A}^{2}\right] .
\end{aligned}
$$

These are plotted in figure 9(a) for a range of $m_{\pi}^{2}$ with $F=92.2 \mathrm{MeV}$ fixed and $g_{A}=1.25$. Note that they are large for the $\left(3_{L}, 5_{R}\right)$ operators already at $m_{\pi} \approx 200 \mathrm{MeV}$. The finite volume correction was also calculated [4]. The corrections ${ }^{2}$ are shown in figure 9(b) for $m_{\pi}=135 \mathrm{MeV}$ as a function of $m_{\pi} L$.

\section{Conclusions}

I discussed three different applications of ChPT that might be useful for lattice calculations.

\footnotetext{
${ }^{2} \mathrm{~A}$ mistake was discovered in the numerical programs used for the plots shown during the conference, so these are different.
} 


\section{Acknowledgements}

This work is supported in part by the Swedish Research Council grants contract numbers 621-20134287, 2015-04089 and 2016-05996 and by the European Research Council (ERC) under the European Union's Horizon 2020 research and innovation programme (grant agreement No 668679).

\section{References}

[1] J. Bijnens, J. Relefors, Vector two-point functions in finite volume using partially quenched chiral perturbation theory at two loops (2017), 1710.04479

[2] J. Relefors, Twisted Loops and Models for Form-factors and the Muon g-2, PhD thesis Lund University, available from http://home.thep.lu.se/ bijnens/publications/thesisjohanr.pdf

[3] J. Bijnens, N. Truedsson Hermansson, The Pion Mass and Decay Constant at Three Loops in Two-Flavour Chiral Perturbation Theory (2017), 1710.01901

[4] J. Bijnens, E. Kofoed, Chiral Perturbation Theory for Neutron-antineutron Oscillations (2017), 1710.04383

[5] E. Kofoed, ChPT for neutron-antineutron oscillations, LU TP 16-62, Master thesis Lund University 2016, available from http://lup.lub.lu.se/student-papers/record/8898924

[6] M. Golterman, Applications of chiral perturbation theory to lattice QCD (2009), 0912 . 4042

[7] J. Bijnens, J. Relefors, JHEP 11, 086 (2016), 1609. 01573

[8] M. Della Morte, A. Juttner, JHEP 11, 154 (2010), 1009. 3783

[9] J. Bijnens, J. Relefors, PoS LATTICE2016, 282 (2016), 1611.06068

[10] C. Aubin, T. Blum, M. Golterman, S. Peris, Phys. Rev. D88, 074505 (2013), 1307.4701

[11] C. Aubin, T. Blum, P. Chau, M. Golterman, S. Peris, C. Tu, Phys. Rev. D93, 054508 (2016), 1512.07555

[12] J. Bijnens, J. Relefors, JHEP 05, 015 (2014), 1402 . 1385

[13] J. Bijnens, K. Ghorbani, Phys. Lett. B636, 51 (2006), hep-lat/0602019

[14] J. Bijnens, G. Ecker, Ann. Rev. Nucl. Part. Sci. 64, 149 (2014), 1405.6488

[15] M. Gell-Mann, R.J. Oakes, B. Renner, Phys. Rev. 175, 2195 (1968)

[16] P. Langacker, H. Pagels, Phys. Rev. D8, 4595 (1973)

[17] J. Gasser, H. Leutwyler, Annals Phys. 158, 142 (1984)

[18] U. Burgi, Nucl. Phys. B479, 392 (1996), hep-ph/9602429

[19] J. Bijnens, G. Colangelo, G. Ecker, J. Gasser, M.E. Sainio, Phys. Lett. B374, 210 (1996), hep-ph/9511397

[20] J. Bijnens, G. Colangelo, G. Ecker, J. Gasser, M.E. Sainio, Nucl. Phys. B508, 263 (1997), [Erratum: Nucl. Phys.B517,639(1998)], hep-ph/9707291

[21] J.A.M. Vermaseren (2000), math-ph/0010025

[22] C. Studerus, Comput. Phys. Commun. 181, 1293 (2010), physics . comp-ph/0912 . 2546

[23] S. Laporta, E. Remiddi, Phys. Lett. B379, 283 (1996), hep-ph/9602417

[24] K. Melnikov, T. van Ritbergen, Nucl. Phys. B591, 515 (2000), hep-ph/0005131

[25] J. Bijnens, L. Carloni, Nucl. Phys. B827, 237 (2010), 0909.5086

[26] J. Bijnens, L. Carloni, Nucl. Phys. B843, 55 (2011), 1008 . 3499

[27] D.G. Phillips, II et al., Phys. Rept. 612, 1 (2016), 1410. 1100

[28] M.I. Buchoff, M. Wagman, Phys. Rev. D93, 016005 (2016), 1506.00647

[29] G. Ecker, M. Mojzis, Phys. Lett. B365, 312 (1996), hep-ph/9508204

[30] V. Bernard, N. Kaiser, U.G. Meissner, Int. J. Mod. Phys. E4, 193 (1995), hep-ph/9501384 